(Kugelberg-Welander disease) and GM2 gangliosidosis are both autosomal recessive in inheritance. Molecular studies on a proband thought to have SMA have uncovered a GM2 gangliosidosis as the cause of the progressive amyotrophy. The SMA gene showed no deletion while the HEXA gene had two mutations. At the onset of GM2 gangliosidosis, clinical manifestations are variable and the differential diagnosis may include SMA or Friedreich ataxia.

\title{
GENETICS OF FRIEDREICH ATAXIA
}

Genotype-phenotype correlations in a group of 100 patients with typical Friedreich ataxia (FRDA), and in three smaller clinically atypical groups (Arcadian FRDA, late-onset FRDA (LOFA), and FRDA with retained reflexes (FARR)), were studied at the Centre de Recherche Louis-Charles Simard, Service de Genetique Medicale, Service de Neurologie, Hopital Sainte-Justine, Departments of Genetics and Medicine, McGill University, Montreal General Hospital, and other centers. Almost all FRDA patients carry a GAA triplet repeat expansion on chromosome 9, despite phenotypic variation. Larger expansions lead to earlier onset and more severe disease. Arcadian FRDA and FARR were unrelated to expansion sizes. (Montermini L, Richter A, Morgan K et al. Phenotypic variability in Friedreich ataxia: role of the associated GAA triplet repeat expansion. Ann Neurol May 1997;41:675-682). (Respond: Dr Massimo Pandolfo, Centre de Recherche Louis-Charles Simard, Pavillon De Seve, 1560 rue Sherbrooke est, Montreal, Quebec, Canada H2L 4M1).

COMMENT. Testing for the GAA expansion is essential for the molecular diagnosis of the various forms of FRDA, and particularly atypical cases of lateonset, those with retained reflexes, and lack of the characteristic hypertrophic cardiomyopathy.

\section{LEUKODYSTROPHY AND OVARIAN DYSGENESIS}

Clinical, biochemical, pathological, and spectroscopic findings in 4 women, aged 15 to 29 years, with leukodystrophy and primary ovarian dysgenesis, are reported from the National Institutes of Neurological Disease, and Child Health and Development, Bethesda, MD. Neurologic evaluations revealed cognitive impairment in 3 (IQs 52,60,77), ataxia at age 10 in one, speech delay in one, febrile seizures in one, neurologic deterioration at ages 610, 17, and 24, mild clumsiness, cerebellar and pyramidal signs, optic atrophy, dysarthria, and facial weakness. Puberty was delayed in 2, and 2 had amenorrhea, with primary gonadal insufficiency and normal hypothalamichypophyseal axis. MRI showed diffuse white matter disease, with frontal cortical atrophy. Proton MRS showed reduction of choline-containing compounds and $\mathrm{N}$-acetylaspartate in the white matter. (Schiffmann R, Tedeschi G, Kinkel RP et al. Leukodystrophy in patients with ovarian dysgenesis. Ann Neurol May 1997;41:654-661). (Respond: Dr Schiffmann, NIH, Bldg 10, Room 3D03, 9000 Rockville Pike, Bethesda, MD 20892).

COMMENT. A novel syndrome of leukodystrophy and primary ovarian dysgenesis of unknown cause is reported in 4 young women who presented with variable neurological impairments in the first or second decades. Chromosomes were normal, and Turner's syndrome was ruled out. Known causes for leukodystrophy were also excluded. The molecular basis for the syndrome remains to be determined. 